\title{
Developed MPPT Algorithm for Photovoltaic Systems without a Voltage Sensor
}

\author{
Milad Momayyezan ${ }^{*}$ and Hossein Iman-Eini ${ }^{\dagger}$ \\ ${ }^{*}$ School of Electrical and Computer Engineering, College of Engineering, University of Tehran, Tehran, Iran
}

\begin{abstract}
This paper presents a study of maximum power point tracking (MPPT) for photovoltaic arrays with only one current sensor. Initially, a review of MPPT methods with only a current sensor is performed with extension for a variety of dc/dc converters. Furthermore, the same topology is developed to achieve better performance in the presence of sensor offset and environmental noise. The proposed method is robust, cost effective, and behaves well dynamically and in the steady state. After a theoretical analysis of presented approach, its validity and effectiveness are verified by simulation and experimental results.
\end{abstract}

Key words: Maximum power point tracking, Power converter, Signal to noise ratio, Solar cells

\section{INTRODUCTION}

In recent years, renewable energy sources, especially photovoltaic (PV) panels and wind turbines have experienced increased attention in terms of power generation. They are pollution-free, reliable and considered to be economical sources of electricity for remote areas. However, the installation costs of PV systems are high [1], and they suffer from low efficiency. Additionally, the nonlinear characteristics of solar cells and their dependency on environmental conditions have a major impact on the complexity of their control techniques [2].

To overcome the above mentioned issues, a variety of MPPT algorithms has been proposed to date. The inherent characteristics of each method make them suitable for particular applications. For instance, [3], [4] present new approaches to solve tracking confusion under rapidly changing climate conditions. Reference [5] makes a comparative study of different MPPT methods in terms of operating conditions, number of sensors, convergence speed, and system cost. Considering above parameters, each method may be considered only for specific applications. According to [6], the Perturb and Observe (P\&O) algorithm has received a great deal of attention due to its simplicity and ease of implementation [7]. A comparative study of low-cost topologies demonstrates the effectiveness of P\&O [8].

Manuscript received Apr. 30, 2013; revised Sep. 7, 2013

Recommended for publication by Associate Editor Trillion Q. Zheng.

†Corresponding Author: imaneini@ut.ac.ir

Tel: +98218208-4962, Fax: + 98218877-8690, University of Tehran

* School of Electrical and Computer Engineering, College of

Engineering, University of Tehran, Iran
Accordingly, a novel algorithm is presented in [9]. The proposed method is implemented by a simple microcontroller, utilizes only one current sensor and has a high overall efficiency [10]. On the other hand, it has been developed only for step-down buck converters and suffers from inherent oscillations around the MPP. According to [11], the boost topology is superior for PV applications with its lower implementation cost and better dynamic response when compared with buck converters. Furthermore, in order to avoid the series connection of PV arrays, it is necessary to boost the input voltage. Therefore, in the first part of this paper, the concepts of the available topologies with only a current sensor are extended for different kinds of $\mathrm{dc} / \mathrm{dc}$ converters. In the second part, the impact of sensor offset and environmental noise on the same methodologies are investigated. Referring to [12], [13], the measurement noise and sensor offset may deteriorate the performance of tracking algorithms. Hence, an analysis of environmental effects and proposing a robust algorithm against noise and offset errors would be a promising solution in practice and for critical applications.

\section{MPPT WITH ONLY ONE CURRENT SENSOR}

The major parameters that affect I-V characteristic of a PV module are temperature and irradiation. Under all conditions, there is only one operating point for a module with the highest power delivery. Using a power converter between the solar module and the load, it is possible to control cell voltage and track the maximum power point. Fig. 1 shows a simplified block diagram of such configuration with the application of a 
boost converter. The output voltage is almost constant due to the connection of battery packs in parallel with the load. Hence, the controller determines the converter duty cycle in a way that maximum power is delivered to the load at different temperature and irradiation levels.

According to (1), the duty cycle represents the switching on-time within each cycle. Eqns. (2)-(4) demonstrate the relation between the cell input voltage, $V_{p v}$ and the load voltage, $V_{\text {bat }}$, in buck, boost, and buck-boost converters, respectively.

$$
\begin{gathered}
D=t_{o n} / T \\
V_{p v}=V_{b a t} / D \\
V_{p v}=V_{b a t} \times(1-D) \\
V_{p v}=V_{b a t} \times(1-D) / D
\end{gathered}
$$

Considering (2)-(4), $V_{p v}$ is written as:

$$
V_{p v}=V_{b a t} \times D^{*}
$$

where, $D^{*}$ is the equivalent of $(1 / D)$ in the buck converter, $(1-D) / D$ in the buck-boost converter, and $(1-D)$ in the boost converter while the converter is operating in the continuous conduction mode (or CCM). Neglecting the system losses, the input power to the converter may be written as:

$$
P_{i n}=V_{p v} \times I_{P v}=V_{b a t} \times\left(D^{*} \times I_{p v}\right)
$$

Since $V_{b a t}$ is almost constant, the following definition is introduced for the equivalent power, $P^{*}$.

$$
P^{*}=I_{p v} \times D^{*}
$$

The principal operation of the $\mathrm{P} \& \mathrm{O}$ algorithm is defined in a way that the cell voltage $V_{p v}$ is a little perturbed and the impact on the delivered power, $P_{i n}$ is continuously observed. This procedure is repeated until condition (8) is achieved.

$$
\partial P_{i n} / \partial V_{p v}=0
$$

Substitution of (7) into (6) and derivation with respect to $V_{p v}$ leads to:

$$
\begin{aligned}
& \partial P_{i n} / \partial V_{p v}=\partial\left[V_{b a t} \times P^{*}\right] / \partial V_{p v} \\
& P^{*} \times \partial\left[V_{b a t}\right] / \partial V_{p v}+V_{b a t} \times \partial\left[P^{*}\right] / \partial V_{p v}=0
\end{aligned}
$$

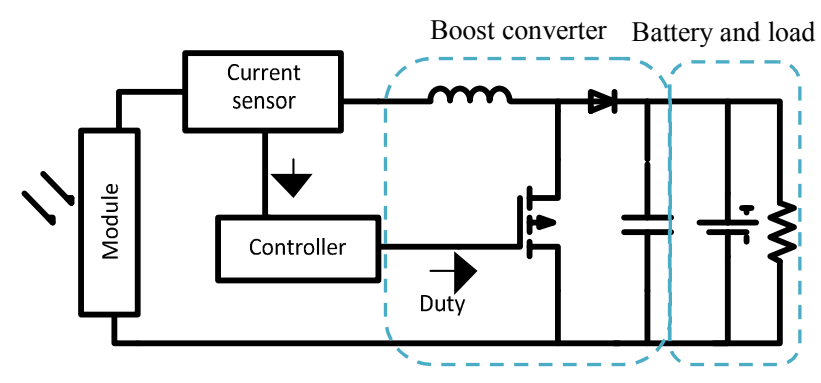

Fig. 1. Application of boost converter as MPPT.

Due to the fact that the battery charging/discharging speed is much lower than the MPPT response, the value of $\frac{\partial v_{\text {bar }}}{\partial v_{p v}}$ is set to zero which yields:

$$
\partial\left[P^{*}\right] / \partial D^{*}=0
$$

In different kinds of $\mathrm{dc} / \mathrm{dc}$ converters, the parameter $D^{*}$ can be determined according to the duty cycle value, $D$. As a result, in general form, the parameter $D$ is perturbed and the equivalent power, $P^{*}$ is observed. Using this approach, only one current sensor is needed to estimate $P^{*}$ while conventional methods need at least two sensors to estimate $P_{\text {in }}$.

\section{IMPROVEMENT OF THE STEADY STATE AND DYNAMIC RESPONSE}

In classic MPPT algorithms, a tradeoff should be made between the speed of the dynamic response and the steady state behavior by appropriate selection of the duty cycle steps. Therefore, in order to achieve a high performance under all conditions, an upadting procedure must be realized. In [14], a modified adaptive method has been proposed for the Hill Climbing approach where the tracker automatically modifies the value of $\Delta D$ to improve the MPPT performance. However, in this paper a simpler algorithm is developed that uses a dynamical variation of $\Delta D$ within the tracking process. Fig. 2 illustrates a flowchart of the proposed method.

According to Fig.2, the duty cycle value $D$ and the step size $\Delta D$ are initialized with $D_{0}$ and $\Delta D_{0}$, respectively. Then, $I_{p v}$ is measured by the current sensor and the paramtere $P^{*}$ is calculated according to (7). In the next step, $\Delta P^{*}=$ $P^{*}(t+\Delta t)-P^{*}(t)$ is computed and compared with the extreme value " $e$ " that defines the acceptable oscillation around the MPP. If the conditional case $\left|\Delta P^{*}\right|>e$ is true, then the duty cycle is updated as follows.

When $\Delta P^{*}$ is positive, the duty cycle is updated with $D_{l}=$ $D_{0}+K \times \Delta D_{0}$, where $K$ is a constant integer, e.g. $K=1$. The negative sign on the other hand, means that the operating 


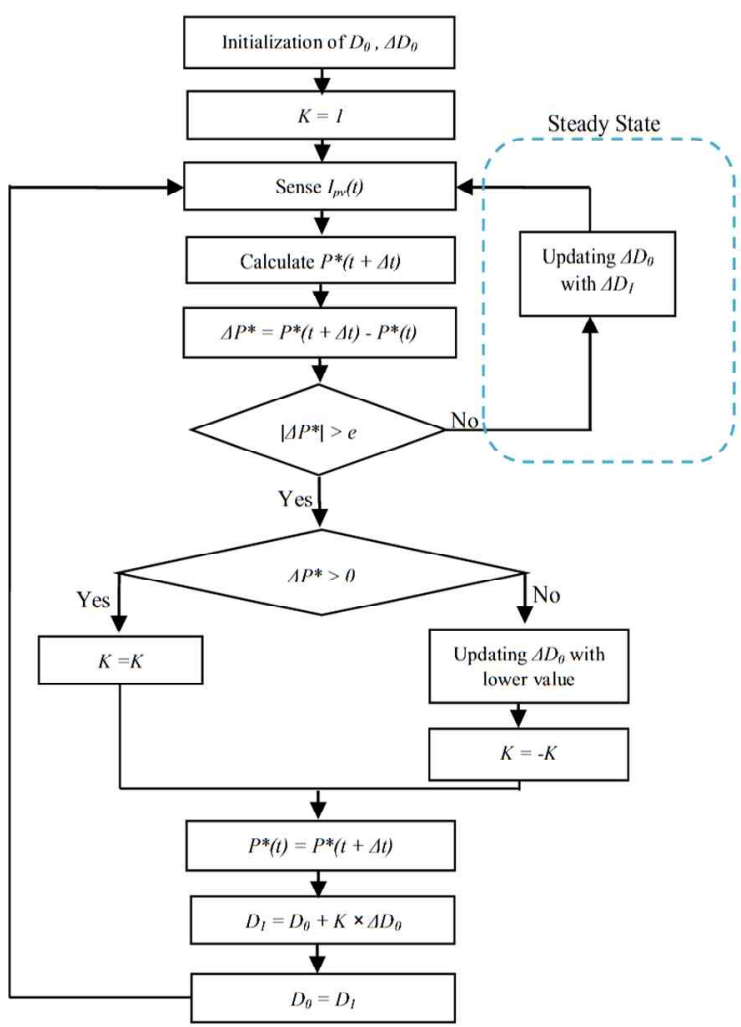

Fig. 2. Algorithm flowchart for presented tracker.

point has already passed the MPP. In this case, $\Delta D$ is reduced by a factor lower than unity for precise tracking of the MPP. The sign of $K$ is then reversed and the value of the duty cycle is tuned for the next cycle.

To achieve fast tracking, the value of $\Delta D$ is chosen to be large enough at initial steps. Whenever $\Delta P^{*}$ becomes negative, the sign of $\Delta D$ is reversed and its value is reduced. This procedure will be repeated until the value of $\left|\Delta P^{*}\right|$ (or the oscillation magnitude around the MPP) becomes smaller than the extreme limit " $e$ ". When the variation of the step size is stopped (or the converter operates at the steady state), it should be able to exit the current situation by the irradiation level or temperature changes. To achieve this goal, the controller updates $\Delta D$ with a larger value at the steady state and waits for environmental changes. Using this strategy, the tracking time, oscillations around the MPP, and the power loss are reduced.

\section{Simulation RESUlts}

To evaluate the behaviour of the proposed algorithm, the PV system shown in Fig. 1 is modelled in the MATLAB/SIMULINK environment [15], based on the configuration shown in Fig. 3. In this case, two parallel modules ("solar module 36/45") supply the load. The characteristic parameters of these modules are given in Table I.



Fig. 3. Simplified solar cell model.

TABLE I

UTILIZED PARAMETERS FOR PV MODULES

\begin{tabular}{ll}
\hline Short circuit current for parallel modules $\left(\mathrm{I}_{\mathrm{sc}}\right)$ & $3.8 \mathrm{~A}$ \\
\hline Series resistance $\left(\mathrm{R}_{\mathrm{s}}\right)$ & $0.503 \Omega$ \\
\hline Open circuit voltage $\left(\mathrm{V}_{\mathrm{oc}}\right)$ & $18 \mathrm{~V}$ \\
\hline Reverse saturation current of the diode $\left(\mathrm{I}_{0}\right)$ & $9.23 \mathrm{nA}$ \\
\hline Number of series cells in a module $(\mathrm{n})$ & 32 \\
\hline Technology coefficient $(\eta)$ & 1.136 \\
\hline Thermal voltage $\left(V_{\mathrm{T}}\right)$ & $26 \times 10^{-3} \mathrm{~V}$
\end{tabular}

The simulation results for the controlled system with the proposed MPPT algorithm are shown in Fig. 4. The represented waveforms are the input power $P_{i n}$, the equivalent power $P^{*}$, the PV current $I_{p v}$, the PV voltage $V_{p v}$, and the corresponding duty cycle as a function of time. The estimated maximum delivered power to the converter was approximately $41 \mathrm{~W}$, while a value of $90 \mathrm{~W}$, according to the module datasheet, was expected. This is due to the fact that the selected parameters, i.e. $I_{s c}=3.8 \mathrm{~A}$ and $V_{o c}=18 \mathrm{~V}$ are chosen based on the real test conditions of Fig. 9(a). In this experiment, the short circuit current and open circuit voltage were far from the ideal condition because of a low irradiation level and cell temperature.

As shown in Fig. 4, the converter duty cycle is increased by the controller from the initial value $(D=0.5)$ to the point in which the maximum power is delivered to the load, i.e. $D=0.59$. It is also obvious that the PV output power $P$ and the defined equivalent power $P^{*}$ demonstrate similar behaviour.

\section{INVESTIGATION OF THE SENSOR OFFSET AND NOISE EFFECT ON MPPT WITH ONLY ONE CURRENT SENSOR}




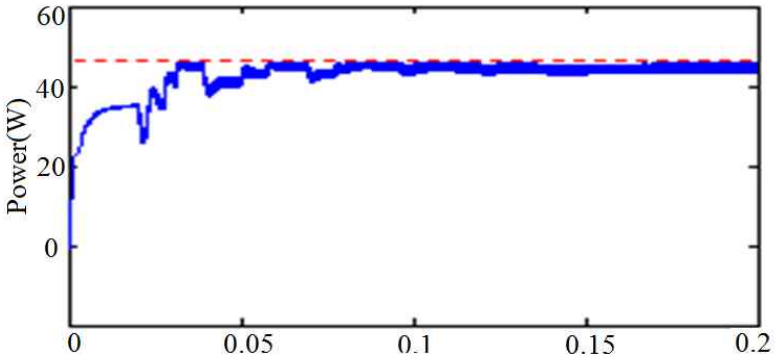

(a)

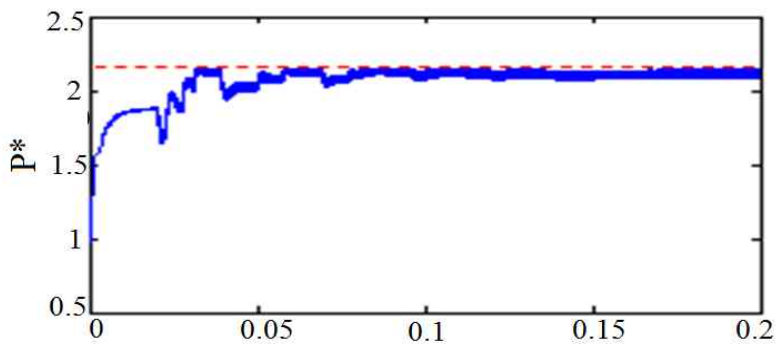

(b)

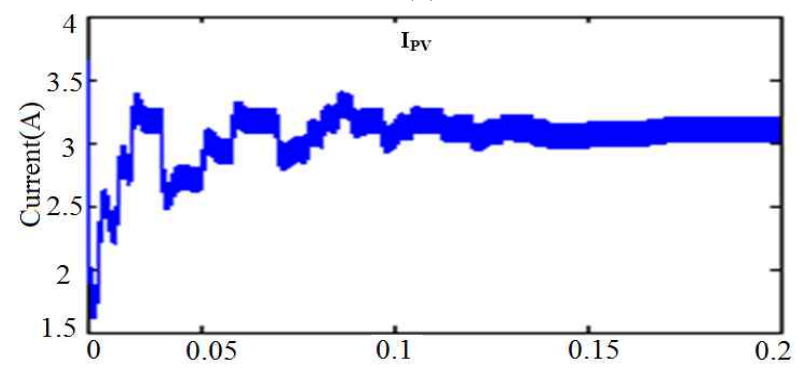

(c)

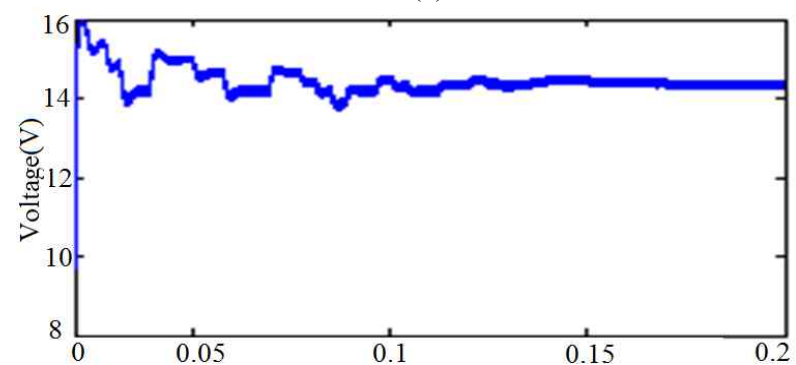

(d)



(e)

Fig. 4. Simulation results for boost converter with improved MPPT algorithm, (a) Output power of PV panels; (b) Defined equivalent power $\mathrm{P}^{*}$; (c) Output current of PV panels; (d) Output voltage of PV panels; and (e) Converter duty cycle versus time.
In this section, the effect of erroneous current measurements on the deviation from the ideal MPP is investigated. As stated in [1], MPPT algorithms typically utilize the cell short circuit current or open circuit voltage to tune the duty cycle and track the MPP. Any erroneous measurements in such approaches result in incorrect MPPT, while the stated procedure with one current sensor overcomes this issue.

First, the error is considered to be a constant deviation (or offset) from the ideal value, which is common in current sensors. Electronic amplifiers and analog-to-digital converters may be sources of dc offset. Fig. 5 shows the evaluation of the tracker in the presence of dc offset.

The module output power has been demonstrated for two different cases: considering the sensor offset and without any offset (or the ideal condition). According to Fig. 5, the sensor offset causes incorrect estimation of the output power. The magnitude of the error depends on the cell operating point and increases by getting closer to the MPP. However, the mentioned issue will not affect the tracker operation since the corresponding duty cycle for MPP (in presence of the dc offset) is equal to the case where the offset equals zero. This advantage simplifies the implementation regarding the $\mathrm{dc}$ offset in measurements.

In the second part, the measurement error is considered to be a random type or (white noise). The tracker suffers from this type of error due to the surrounding environment, dc power supplies or the switching action of power converters. Like many conventional methods such as $\mathrm{P} \& \mathrm{O}$ and Hill Climbing, the presented method uses a differential term, i.e. $\Delta P^{*}$, to track the MPP. However, the application of differential terms will result in a low signal to noise ratio (or SNR) and a deterioration of tracker.

The following simulation evaluates the performance of $\mathrm{P} \& \mathrm{O}$ and the suggested method in the presence of white noise. In this simulation, White Gaussian Noise is added deliberately to the sensed current. The corresponding result for a noise with a power of $-35 \mathrm{db}$ is demonstrated in Fig. 6.

As illustrated in Fig. 6, both algorithms, which are implemented based on differential terms, are not accurate enough and may fail. Dark circles 1 and 2 highlight the deviation of the tracker during transient and steady state conditions, respectively. Such deteriorations lead to higher losses and weak performance in real conditions. As an alternative, a partial derivative expression is used instead of differential terms. Although this modification adds some complexity, it may be a solution to reduce the noise effect and to improve the steady state performance. In addition, because of accurate MPP detection, there is no oscillation around the MPP which implies higher efficiency. 


\section{Proposing A Robust Algorithm FOR NOISY ENVIRONMENTS}

As stated in section 2, the following equation may be employed for tracking the MPP in different kinds of $\mathrm{dc} / \mathrm{dc}$ converters:

$$
\frac{\partial P^{*}}{\partial D}=0
$$

Inserting the value of $P^{*}$ according to (7) into (11), yields:

$$
\frac{\partial\left(I_{p v} \times D^{*}\right)}{\partial D}=0
$$

Substituting the value of $D^{*}$, e.g. for the boost converter in $\mathrm{CCM}$, leads to the following condition:

$$
-I_{p v}+\frac{(1-D) ? \partial_{p v}}{\partial D}=0
$$

To solve the differential equation of (13), the partial term $\frac{\partial I_{n v}}{\partial D}$ is rephrased. In this case, first $I_{p v}$ is calculated by substitution of the following relations (derived from Fig. 3 and equation (4)) into (13):

$$
\begin{gathered}
I_{D}=I_{s}-I_{p v} \\
V_{D}=V_{p v}+R_{s} \times I_{p v} \\
V_{p v}=(1-D) \times E_{b a t}
\end{gathered}
$$

Then $I_{p v}$ is written as:

$$
I_{p v}=I_{s}-m I_{0} \exp \left(\frac{V_{p v}+R_{s} \times I_{p v}}{n \eta V_{T}}\right)
$$

As a result, the following relation for $\frac{\partial I_{M V}}{\partial D}$ is derived:

$$
\frac{\partial I_{p v}}{\partial D}=-m I_{0}\left[\frac{-E_{b a t}+R_{S} \frac{\partial I_{p v}}{\partial D}}{n \eta V_{T}}\right] \times \exp \left(\frac{V_{p v}+R_{S} I_{p v}}{n \eta V_{T}}\right)
$$

Or:

$$
\frac{\partial I_{p v}}{\partial D}=\frac{\left(m I_{0} E_{b a t}\right) \times \exp \left(\frac{(1-D) E_{b a t}+R_{S} I_{p v}}{n \eta V_{T}}\right)}{\left(m I_{0} R_{S}\right) \times \exp \left(\frac{(1-D) E_{b a t}+R_{S} I_{p v}}{n \eta V_{T}}\right)+n \eta V_{T}}
$$

Substituting (19) into (13) leads to (20) which is a mathematical equation without any differential terms.

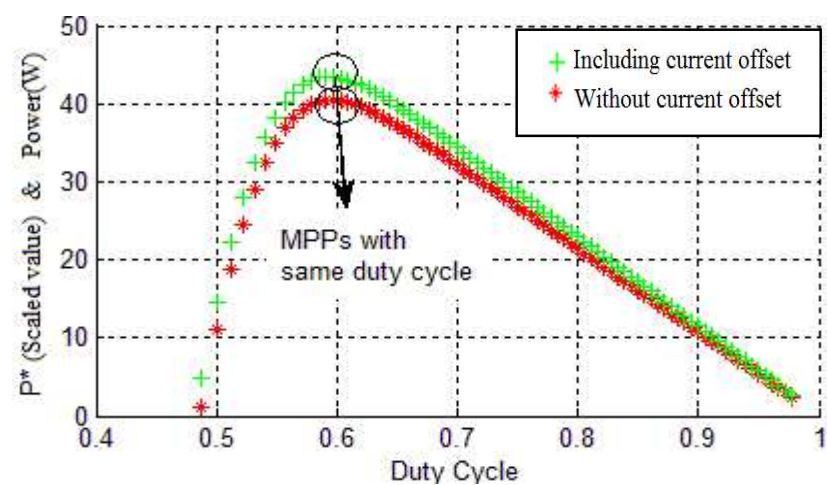

Fig. 5. Evaluation of MPPT algorithm in presence of sensor offset
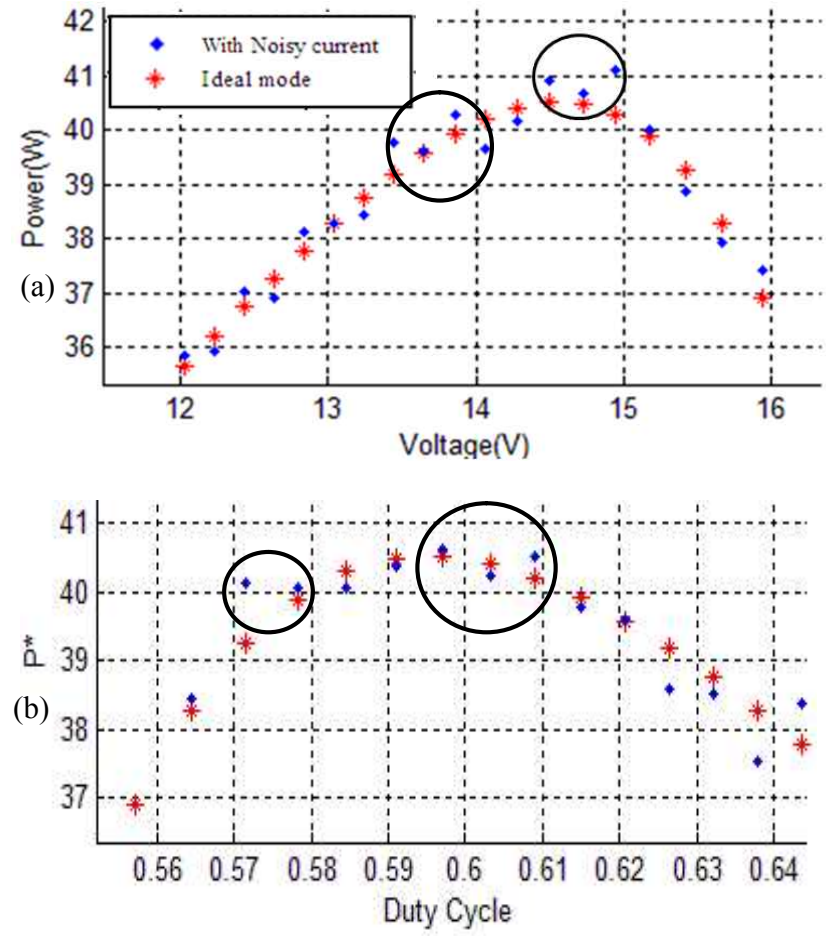

Fig. 6. Evaluation of noise effect on MPPT algorithm, (a) P\&O algorithm; (b) suggested algorithm with one current sensor.

$$
R E F=I_{p v}-(1-D) \frac{\left(m I_{0} E_{b a t}\right) \exp \left[\frac{(1-D) E_{b a t}+R_{s} I_{p v}}{n \eta V_{T}}\right]}{\left(m I_{0} R_{S}\right) \exp \left[\frac{(1-D) E_{b a t}+R_{s} I_{p v}}{n \eta V_{T}}\right]+n \eta V_{T}}=0
$$

The parameter $R E F$ is used to represent the value of $\frac{\partial P^{\prime}}{\partial D}$. According to (20), the tracker should modify the duty cycle $D$ and measures the current $I_{p v}$ until the conditional case " $R E F=$ 0 " is achieved. In practice, reaching this condition is not applicable due to the discontinuity of the duty cycle. Therefore, the best operating point is where the magnitude of $R E F$ becomes minimum (as close as possible to zero). It 


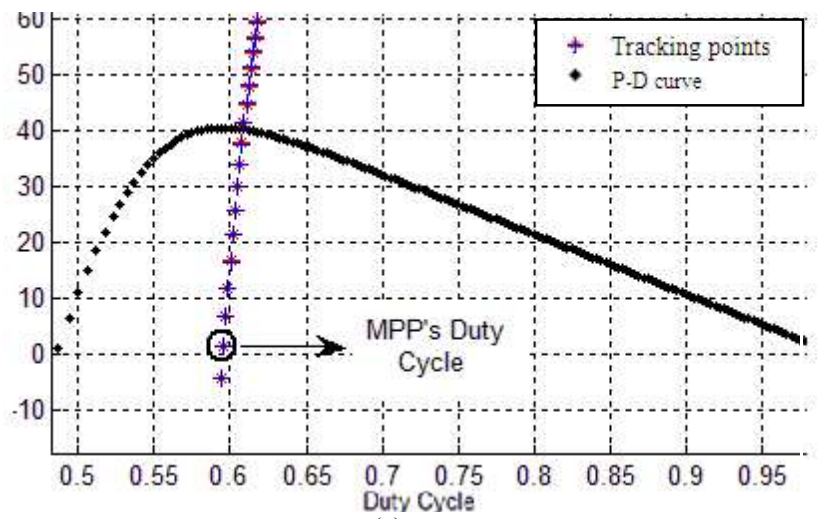

(a)

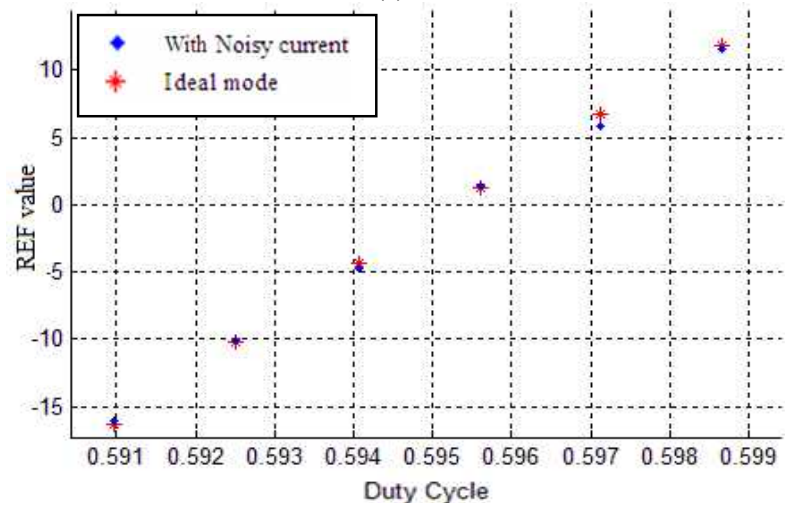

(b)

Fig. 7. Investigation of proposed MPPT algorithm based on eq. (20), (a) Module output power and the value of $R E F$ as a function of duty cycle; (b) Tracking points around MPP with and without considering white noise.

should be noted that (20) is valid for the boost converter while it can be recalculated for typical $\mathrm{dc} / \mathrm{dc}$ converters in a similar way.

\section{INVESTIGATION OF A ROBUST ALGORITHM BY SIMULATION}

To evaluate the performance of the proposed method, the last simulation is repeated in the presence of white noise. In this case, the module output power and the value of $R E F$ (or $\left.\frac{\partial P^{*}}{\partial D}\right)$ are demonstrated as a function of the duty cycle. Fig. 7 shows the simulation results while the system is asymptotically stable and any variations in the duty cycle at the steady state will be returned to the MPP by the tracker.

According to Fig. 7(a), the maximum power point corresponds to a duty cycle in which the value of $R E F$ is almost zero. Fig. 7(b) demonstrates the robustness of the proposed algorithm in terms of noisy current where tracking points are shown for two cases: considering white noise (blue points) and without noise (red points). Comparing the obtained results with those in Fig. 6(a)-(b), the robustness of the tracker is confirmed. In the presented method with the

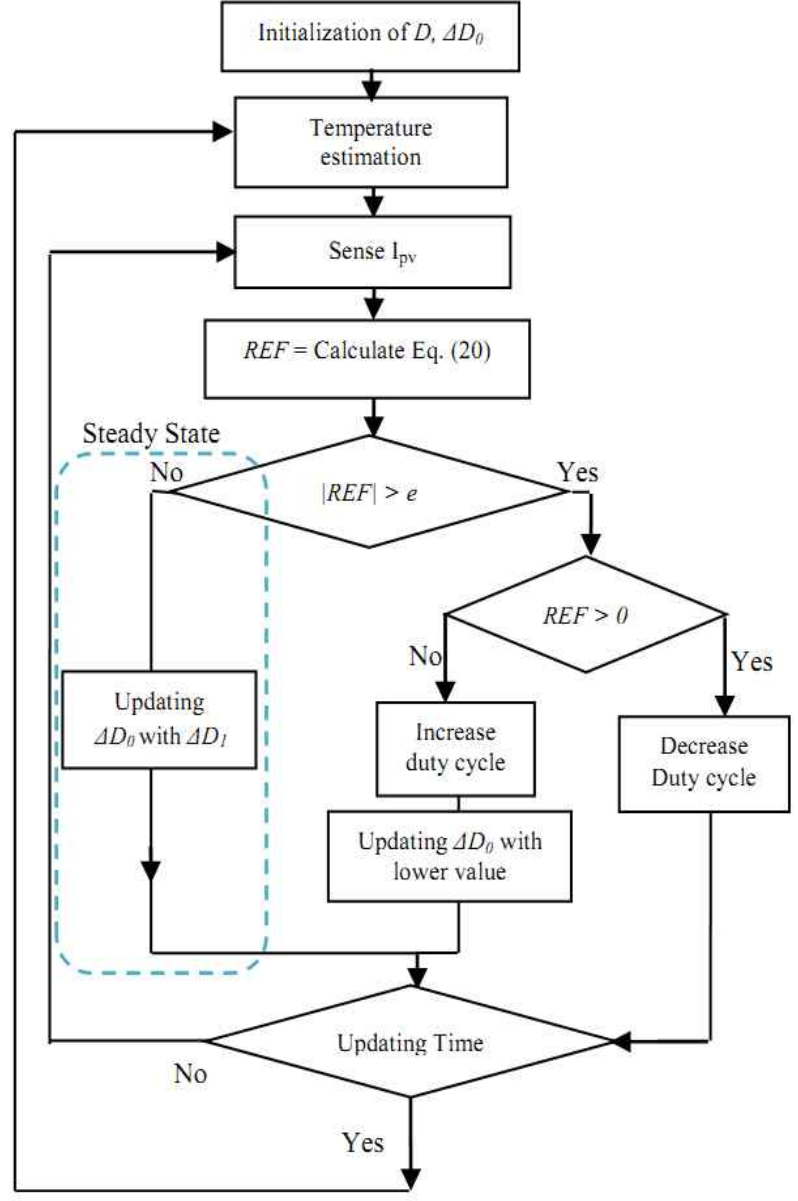

Fig. 8. Flowchart of new MPPT algorithm.

concept of eq. (20), the parameters dependent on module temperature may affect the efficiency of the PV system, [16-17]. Hence, for successful MPPT under all conditions, the effects of temperature variations on $V_{T}$ and $I_{0}$ need to be considered. Equations (21) and (22) describe the dependency of $I_{0}$ and $V_{T}$ on temperature, respectively.

$$
\begin{gathered}
I_{0}=I_{0 N}\left(T / T_{N}\right)^{3} \exp \left(\frac{E_{g}}{\eta k / q}\left(\frac{1}{T_{N}}-\frac{1}{T}\right)\right) \\
V_{T}=\frac{k T_{N}}{q} \times \frac{T}{T_{N}}=V_{T N} \times \frac{T}{T_{N}}
\end{gathered}
$$

where, $I_{0 N}$ and $V_{T N}$ are the saturation current and thermal voltage at standard temperature $T_{\mathrm{N}}=300^{\circ} \mathrm{k}$. $E_{g}$ is the silicon band gap energy, $k$ is the Boltzmann constant, and $q$ is the electric charge of the electron. Referring to the literature, valuable contributions towards the model based methods for estimating the temperature of PV modules are available in [17-19]. In this paper, the NOCT model described in [17] is employed to estimate the cell temperature. The MPPT algorithm with the improved characteristics of being insensitive to noise, dc offset, and converter topology is shown in Fig. 8. In the beginning, the cell temperature and 


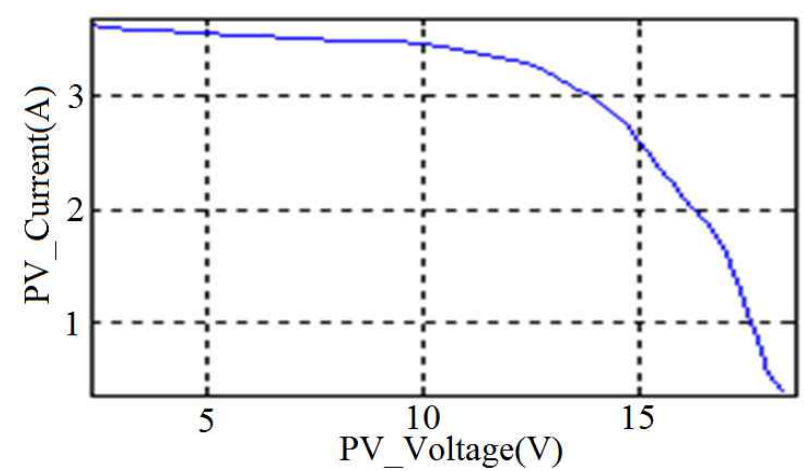

(a)



(b)

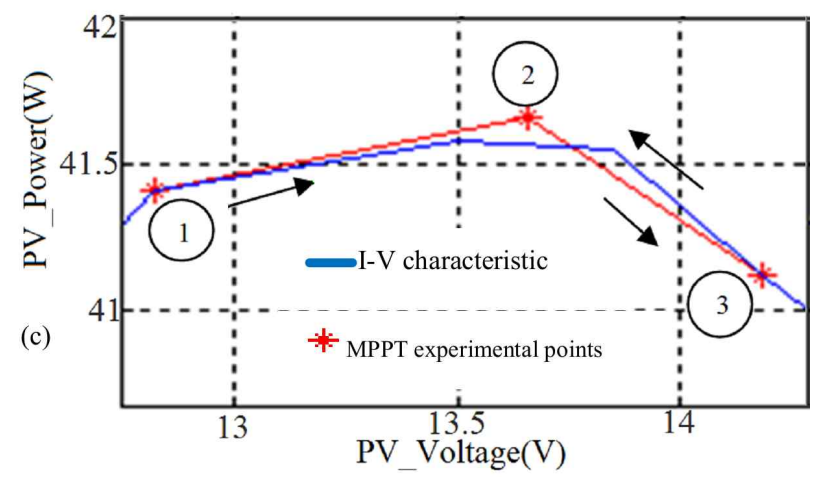

(c)

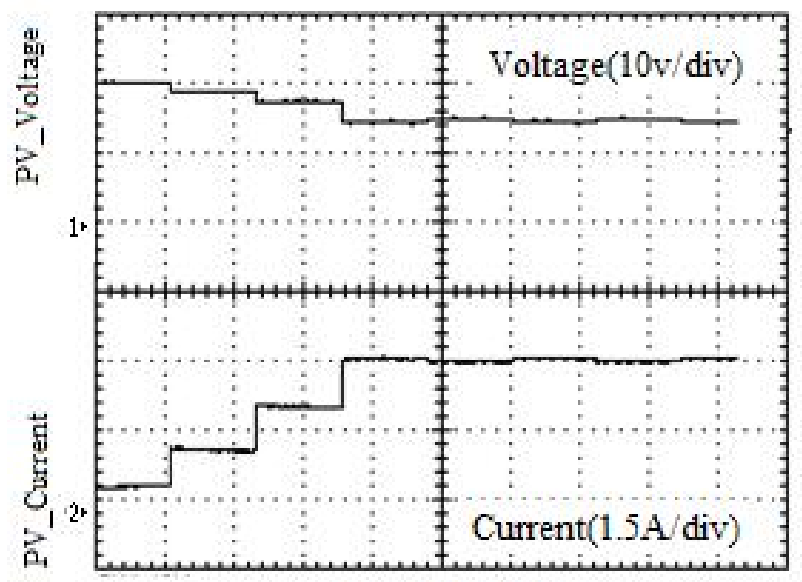

Time $(0.5 \mathrm{~s} / \mathrm{div})$

(d)

Fig. 9. Evaluation of proposed MPPT algorithm at 1:30 PM, (a) I-V characteristic of two parallel modules; (b) P-V characteristic along with tracking points; (c) three final points around MPP; and (d) PV voltage and PV current waveforms during MPP tracking. the PV current $I_{p v}$ are measured. Then, the mathematical expression of (20) is calculated as a reference (termed as REF). This value is compared to the extreme parameter " $\mathrm{e}$ ", which defines an acceptable deviation from the MPP. Then, the value of the duty cycle is updated within each period until the conditional case is satisfied. Like the explained algorithm in Fig. 2, the duty cycle steps are reduced whenever the tracker passes the MPP. Moreover, to exit from the steady state in the case of environmental changes, the tracker looks for variations and modifies the parameter $\Delta D$ with a larger step size. The estimation of the cell temperature will be performed with a lower frequency due to the slower dynamics of temperature variations.

In order to analyse the performance of the explained algorithm, a setup similar to the structure in Fig. 1 has been implemented. The hardware setup includes two parallel modules ("solar module 36/45"), a low cost microcontroller, a boost converter, and three paralleled $60 \mathrm{Ah}-12 \mathrm{~V}$ lead-acid batteries with a resistive load. Fig. 11 shows an image of the hardware setup.

\section{EXPERIMENTAL RESULTS}

The first experiment was carried out at around 1:30 PM. Fig. 9(b-d) show the tracking process of a controller based on the presented MPPT algorithm.

As shown, the first three steps are larger to achieve a fast dynamic response. Then, the duty cycle step reduces to minimize the oscillations around the MPP and to improve the steady state performance. Fig. 9(c) shows the last three points that the controller oscillates between. It should be noticed that the oscillation margin may be reduced more by decreasing the extreme value "e". The variations of the PV voltage and current during the tracking process are shown in Fig 9(d). According to this figure, the tracker found the MPP after 4 iterations (or 2 seconds) from system start up. As time passes, the voltage and current variations around the MPP reduce and consequently the power oscillations become lower.

The last experiment investigates the performance of the proposed algorithm under different conditions. This test was performed at around 6:30 PM where the irradiation level was lower. The results are shown in Fig.10. It should be noted that the tracking is processed in existence of the dc offset from the current sensor.

Because of air pollution and air dust, the I-V characteristics of the solar panels were different from the ideal condition. According to the cell datasheet, the short circuit current for each module should be around 2.98A. However, in practice the maximum short circuit current was $2 \mathrm{~A}$. The measured I-V characteristics for the two parallel modules just before testing and for two different hours of a summer day are illustrated in Fig. 9(a) and 10(a). 

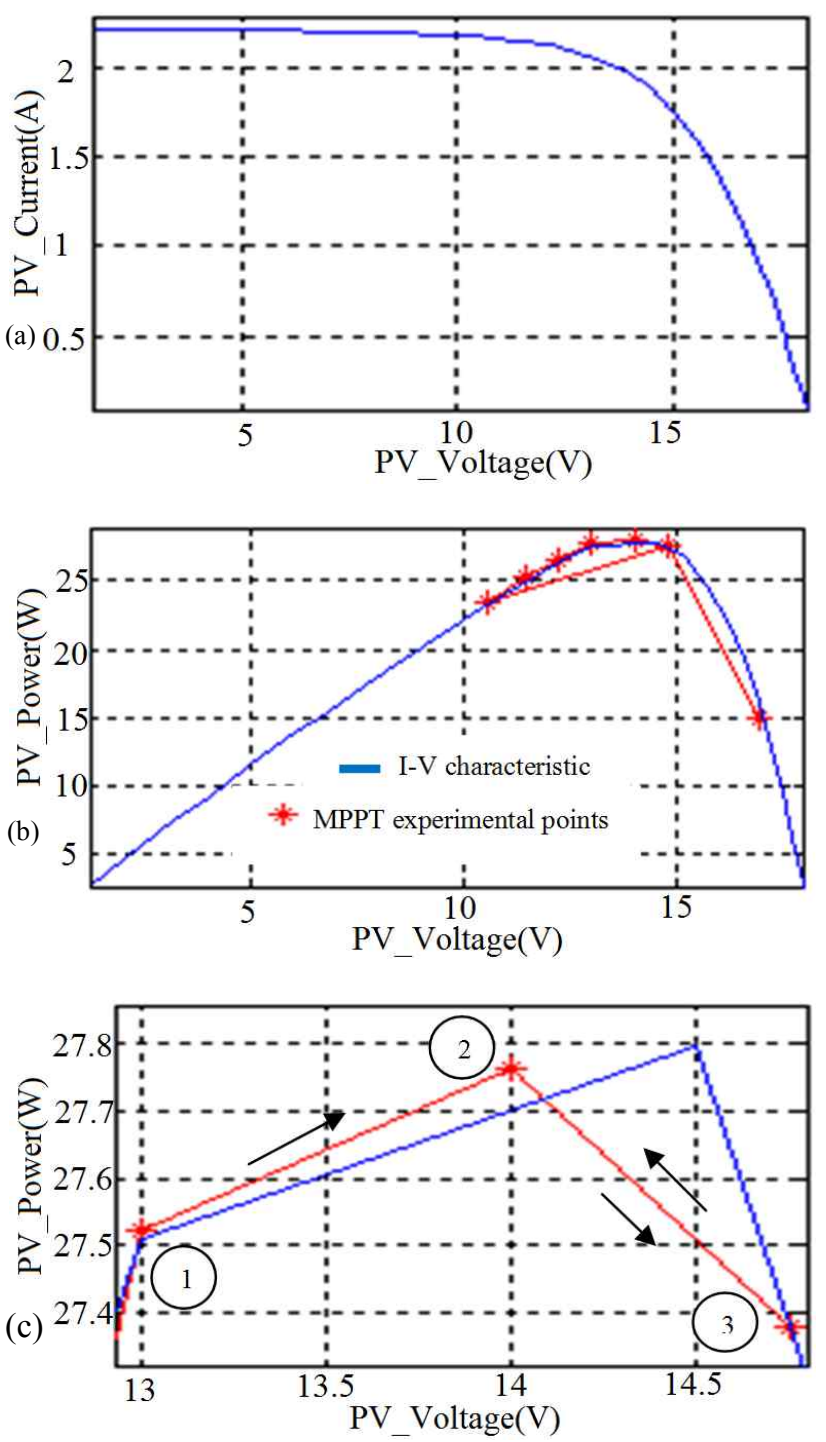

Fig. 10. Evaluation of proposed MPPT algorithm at 6:30 PM, (a) I-V characteristic of two parallel modules; (b) P-V characteristic along with tracking points; and (c) three final points around MPP.

\section{CONCLUSIONS}

In this paper, after studying conventional algorithms that utilize only one current sensor, a novel MPPT method is proposed. The major characteristics of the presented algorithm are robustness against environmental noise with acceptable performance in the steady state and under transient conditions. Furthermore, it may be implemented for different kinds of dc/dc converters with the application of a simple and low-cost microcontroller. To improve the dynamic response and minimize the oscillations around the MPP, variable step size method is employed. The validity and performance of the new method are verified by simulation and experimental results.

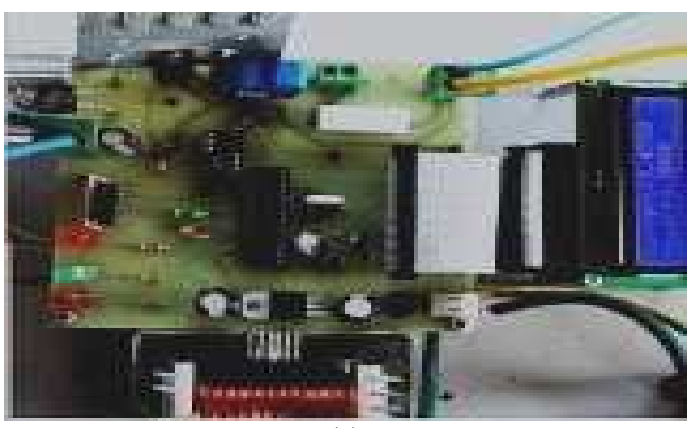

(a)

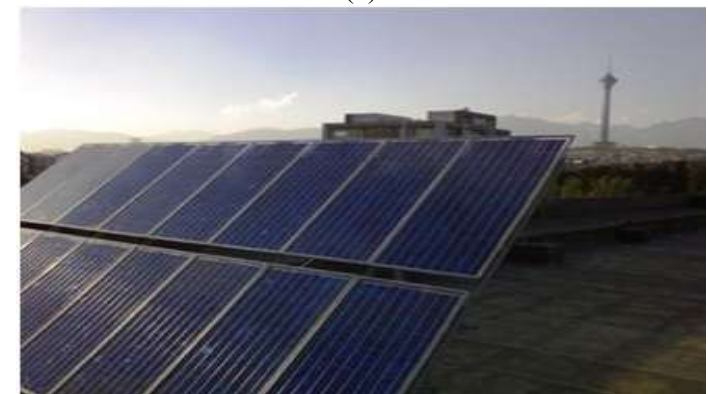

(b)

Fig. 11. Experimental setup, (a) boost converter with controller; (b) "36/45 solar modules" employed in the experiment.

\section{REFERENCES}

[1] J. H. R. Enslin, M. S. Wolf, D. B. Snyman, and W. Swiegers, "Integrated photovoltaic maximum power point tracking converter," IEEE Trans. Ind. Electron., Vol. 44, No. 6, pp. 769-773, Dec. 1997.

[2] H. Patel and V. Agarwal, "Maximum power point tracking scheme for PV systems operating under partially shaded conditions," IEEE Trans. Ind. Electron., Vol. 55, No. 4, pp.1689-1698, Apr. 2008.

[3] D. Sera, R. Teodorescu, J. Hantschel and M. K.noll, "Optimized maximum power point tracker for fast-changing environmental conditions," IEEE Trans. Ind. Electron., Vol. 55, No. 7, pp. 2629 - 2637, Jul. 2008.

[4] N. Fermia, D. Granozio, G. Petrone, and M. Vitelli, "Predictive \& adaptive MPPT perturb and observe method," IEEE Trans. Aerosp. Electron. Syst., Vol. 43, No. 3, pp. 934-950, Jul. 2007.

[5] T. Esram and P. L. Chapman, "Comparison of photovoltaic array maximum power point tracking techniques," IEEE Trans. Energy Convers., Vol. 22, No. 2, pp. 439-449, Jun. 2007.

[6] V. Salas, E. Olías, A. Barrado, A. Lázaro, "Review of the maximum power point tracking algorithms for stand-alone photovoltaic systems," Solar Energy Materials \& Solar Cells, Vol. 90, No. 11, pp. 1555-1578, Jul. 2006.

[7] N. Femia, G. Petrone, G. Spagnuolo, and M. Vitelli, "Optimization of perturb and observe maximum power point tracking method," IEEE Trans. Power Electron., Vol. 20, No.4, pp. 963- 973, Jul. 2005.

[8] D. P. Hohm and M. E. Ropp, "Comparative study of maximum power point tracking algorithms," Journal of Progress in Photovoltaics: Research and Applications, Wiley Interscience, Vol. 11, No. 1, pp. 47-62, 2003. 
[9] V. Salas, E. Olías, A. Lázaro, and A. Barrado, "New algorithm using only one variable measurement applied to a maximum power point tracker," Solar Energy Materials and Solar Cells, Vol. 87, No. 1-4, pp. 675-684, May 2005.

[10] V. Salas, E. Olias, A.Lazaro, and A. Barrado, "Evaluation of a new maximum power point tracker (MPPT) applied to the photovoltaic stand-alone systems," Solar Energy Materials \& Solar Cells, Vol. 87, No. 1-4, pp. 807-815, May 2005

[11] W. Xiao, N. Ozog, and W. G. Dunford, "Topology study of photovoltaic interface for maximum power point tracking," IEEE Trans. Ind. Electron, Vol. 54, No. 3, pp. 1696-1704, Jun. 2007.

[12] H. Al-Atrash, I. Batarseh, and K. Rustom, "Effect of measurement noise and bias on Hill-Climbing MPPT algorithms," IEEE Trans. Aerosp. Electron. Syst., Vol. 46, No. 2, pp. 745-760, Apr. 2010.

[13] A. Latham and C. Sullivan, "Optimization of a continuous-time maximum power point tracking algorithm in the presence of noise," in IEEE Workshop on Control and Modeling for Power Electronics, 2010.

[14] W. Xiao and W. G. Dunford, "A modified adaptive hill climbing MPPT method for photovoltaic power systems," in Proc. 35th Annu. IEEE Power Electron. Spec. Conf., pp. 1957-1963, 2004

[15] G. Walker, "Evaluating MPPT converter topologies using a MATLAB PV model," J. Electr. Electron. Eng., Vol. 21, No. 1, pp. 49-56, 2001.

[16] K. Nishioka, T. Hatayama, Y. Uraoka, T. Fuyuki, R. Hagihara, and M. Watanabe, "Field-test analysis of PV-system-output characteristics focusing on module temperature," Journal of Solar Energy Materials and solar cells, Vol. 75, No. 3, pp. 665-71, 2003.

[17] M. Mattei, G. Notton, C. Cristofari, M. Muselli, and P. Poggi, "Calculation of the polycrystalline PV module temperature using a simple method of energy balance," Renewable Energy. Vol. 31, No. 4, pp. 553-567, Apr. 2006.
[18] D. L. King, W. E. Boyson, and J. A. Kratochvil, "Photovoltaic Array Performance Model," Sandia National Lab., SAND2004-3535, Aug. 2004.

[19] H. A. Krishna, N. K. Misra and M. S. Suresh. "Solar cell as a temperature sensor for measuring temperature of solar panels in Satellites," Journal of Instrum. Soc. of India, Vol. 39, No. 1, Mar. 2009.

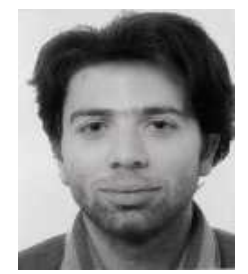

Milad Momayyezan was born in Isfahan, Iran, in 1988. He received his B.S. in Electrical Engineering from the University of Tehran, Iran, in 2011. In the same year, he started his M.S. education at the Royal Institute of Technology (KTH), Stockholm, Sweden. He is currently with the Institute for Power Electronics and Electrical Drives (ISEA), Aachen, Germany. His current research interests include renewable energy systems, power convertors, and electric vehicles.

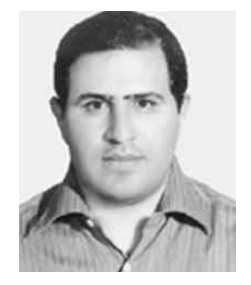

Hossein Iman-Eini received his B.S. and M.S. from the University of Tehran, Tehran, Iran, in 2001 and 2003, respectively, and his $\mathrm{Ph} . \mathrm{D}$. from both the University of Tehran and the Grenoble Institute of Technology, Grenoble, France, in 2009, all in Electrical Engineering. He is currently an Assistant Professor in the School of Electrical and Computer Engineering, University of Tehran. His current research interests include the modeling and control of power converters, multilevel converters and renewable energy systems. 